\title{
Long non-coding RNA Irm enhances myogenic differentiation by interacting with MEF2D
}

\author{
Yutong Sui', Yu Han², Xingyu Zhao ${ }^{2}$, Dongsong $\mathrm{Li}^{2}$ and Guangyu $\mathrm{Li}^{1}$
}

\begin{abstract}
Recent studies suggest important roles for long non-coding RNAs as essential regulators of myogenic differentiation. Here, we report that IncRNA Irm is upregulated during myogenesis. Functional analyses show that the overexpression of Irm enhances myogenic differentiation, whereas the inhibition of Irm has completely opposite effects in vitro. Notably, the inhibition of Irm blocks damage-induced muscle regeneration in vivo. Mechanistically, Irm regulates the expression of myogenic genes by directly binding to MEF2D, which in turn promotes the assembly of MyoD/MEF2D on the regulatory elements of target genes. Collectively, we have identified a novel IncRNA that interacts with MEF2D to regulate myogenesis.
\end{abstract}

\section{Introduction}

Long non-coding RNAs (lncRNAs) are a class of transcripts of more than 200 nucleotides without proteincoding potential. Within the last few years, thousands of IncRNAs have been identified and shown to play pivotal roles in numerous important biological processes, including cellular proliferation ${ }^{1}$, differentiation ${ }^{2}$ and development $^{3}$, chromosomal imprinting ${ }^{4}$, and genomic stability ${ }^{5}$. Worth to note, several IncRNAs have been determined to regulate myogenesis. For example, IncRNA AK017368 promotes the proliferation and suppresses the differentiation of myoblasts in skeletal muscle development by attenuating the function of miR-30c ${ }^{6}$. IncRNA Dum interacts with Dnmts to regulate Dppa2 expression during myogenic differentiation and muscle regeneration ${ }^{7}$. Lnc-mg promotes myogenesis, by functioning as a competing endogenous RNA for microRNA-125b to control the

Correspondence: Dongsong Li (lidongsong@jlu.edu.cn) or

Guangyu Li (liguangyu@caas.cn)

${ }^{1}$ State Key Laboratory for Molecular Biology of Special Economic Animals,

Institute of Special Animal and Plant Sciences, Chinese Academy of Agricultural Sciences, Changchun 130112, China

2Joint Surgery Department, No.1 Hospital of Jilin University, Changchun

130021, China

Edited by Candi protein abundance of insulin-like growth factor $2^{8}$. However, the amount of characterized lncRNAs that regulate myogenesis is merely the tip of the iceberg, and a large number of lncRNAs remain to be characterized.

Myogenesis is a highly coordinated developmental process that contributes to the formation and maintenance of muscle tissue. Myogenic cell specification and differentiation are controlled by a complex network of myogenic regulatory factors, including MyoD (myogenic differentiation), muscle bHLH proteins Myf5, myogenin (MyoG), and MEF2 family ${ }^{9-11}$. Recent studies have indicated various molecular mechanisms for lncRNAs and the current best characterized is in the regulation of epigenetic dynamics and gene expression ${ }^{12}$. Indeed, some muscle-specific lncRNAs that control muscle gene expression have been reported, including lncRNA $\mathrm{Dum}^{7}$, muscle-specific linc-MD1 ${ }^{13}$, linc-RAM ${ }^{14}$, and $\operatorname{Linc}-Y Y 1^{15}$. lncRNA Irm has been previously identified as an alternatively splicing isoform of Rian gene ${ }^{16}$. Worth to note, a recent study has suggested that Irm was associated with the morphogenesis of skeletal muscle during embryonic development, indicating its pivotal role in myogenesis ${ }^{17}$. However, the biological function of Irm in the development of skeletal muscle remains unclear.

\section{(c) The Author(s) 2019}

(c) (i) Open Access This article is licensed under a Creative Commons Attribution 4.0 International License, which permits use, sharing, adaptation, distribution and reproduction cc) in any medium or format, as long as you give appropriate credit to the original author(s) and the source, provide a link to the Creative Commons license, and indicate if changes were made. The images or other third party material in this article are included in the article's Creative Commons license, unless indicated otherwise in a credit line to the material. If material is not included in the article's Creative Commons license and your intended use is not permitted by statutory regulation or exceeds the permitted use, you will need to obtain permission directly from the copyright holder. To view a copy of this license, visit http://creativecommons.org/licenses/by/4.0/. 
Here, we examined the functional role of Irm in the development of skeletal muscle. We showed that the expression of Irm is tightly associated with myogenic processes in vitro and in vivo. Furthermore, functional studies demonstrated that it acts as a pro-myogenic factor in both myoblast differentiation and muscle regeneration. Mechanistically, we revealed that Irm regulates the transcription of myogenic genes by directly binding to MEF2D, which in turn promotes the assembly of the MyoD-MEF2D complex on the regulatory elements of target genes.

\section{Results}

\section{LncRNA Irm is associated with skeletal myogenesis}

Recent studies have shown that Irm is associated with the morphogenesis of skeletal muscle during embryonic development ${ }^{17}$. Therefore, we hypothesized that Irm may also be involved in myogenesis. To investigate its relevancy in myogenesis, we examined its temporal and spatial expression patterns in several myogenesis systems in vitro and in vivo. First, the $\mathrm{C} 2 \mathrm{C} 12$ cells were shifted to Dulbecco's modified Eagle's medium (DMEM) containing $2 \%$ horse serum for myogenic differentiation experiment (Fig. 1a). We found that the expression of MyoD and myogenin was significantly increased during the differentiation of $\mathrm{C} 2 \mathrm{C} 12$ cells (Fig. 1a). Meanwhile, the expression of Rian had no change during the differentiation of $\mathrm{C} 2 \mathrm{C} 12$ cells (Fig. 1b). However, Irm was found to be significantly upregulated during the stage from day 0 to day 3 in the differentiation medium but gradually decreased afterwards (day 5) (Fig. 1c), suggesting that it can be a myogenic factor during differentiation. Furthermore, the primary myoblasts, which were isolated from 10-day-old mouse muscles, were shifted to the differentiation medium for myogenic differentiation experiment (Fig. 1d, e). Consistently, the expression of MyoD and myogenin was significantly increased during primary myoblast differentiation. Meanwhile, the kinetics of Rian and Irm expression was also confirmed during the differentiation of freshly isolated primary myoblasts (Fig. 1f, g). In addition, we examined the expression dynamics of Irm during myogenesis in vivo. By employing a cardiotoxin (CTX)-induced muscle regeneration model, we found that Irm is highly induced during the regeneration stage (Fig. 1h, i). Consistently, high levels of Irm were observed in the limb muscles of newborn mice (at the age of 3 days and 8 days), which displayed active myogenesis, but the level of Irm decreased as the neonatal myogenesis ceased after about 2 weeks and remained low as the mice aged (Fig. 1j). These results indicated that Irm is associated with active myogenesis in vitro and in vivo.

\section{Irm promotes myogenic differentiation}

Given the induction of $\mathrm{Irm}$ expression during $\mathrm{C} 2 \mathrm{C} 12$ differentiation, it was conceivable that Irm plays a regulatory role in regulating myogenesis. Thus, we first examined the effect of Irm on the myogenic differentiation of $\mathrm{C} 2 \mathrm{C} 12$ cells (Fig. 2a). The overexpression of Irm resulted in a significant increase in $\mathrm{C} 2 \mathrm{C} 12$ cell differentiation as proved by the increased myosin heavy chain (MHC) immunostaining (Fig. 2b), increased fusion index (Fig. 2b), and the upregulated expression of myogenic marker genes myogenin and MHC (Fig. 2c-f). Conversely, the knockdown of Irm by two independent small interfering RNAs (siRNAs) (Fig. 3a) inhibited the differentiation of $\mathrm{C} 2 \mathrm{C} 12$ cells with reduced $\mathrm{MHC}$ immunostaining (Fig. 3b), decreased fusion index (Fig. 3b), and downregulated myogenin and $\mathrm{MHC}$ expression (Fig. 3c-f). Taken together, all these results indicated that Irm promotes myogenic differentiation.

\section{Loss of Irm delayed CTX-induced muscle regeneration in vivo}

The above findings underscored the role of Irm in myoblast differentiation, suggesting that it may play a role in muscle regeneration in vivo. To this end, we depleted Irm in mouse limb muscles during injuryinduced regeneration. As illustrated in Fig. 4a, an injection of si-Irm-1 and si-scramble was performed two times on days 1 and 4 post CTX injection and muscles were harvested at the designated times for analyses. We found that the injection of si-Irm-1 led to a significant loss of Irm expression during the time course (Fig. 4b, c), as detected by RNA fluorescence in situ hybridization (RNA-FISH) and quantitative real-time polymerase chain reaction (qRT-PCR) assays. Consistently, the expression levels of myogenin were significantly decreased (Fig. 4d, e), as detected by western blotting and qRT-PCR assays. CTX induced extensive muscle damage in both si-Irm-1-group and si-scramblegroup muscles on the day after injury (Fig. 4f). Regenerating myofibers, which are characterized by centralized nuclei, were significantly smaller in si-Irm-1 groups than in si-scramble groups at 6 days after CTX injection (Fig. 4f, g), with an average size of $371.5 \pm 36.4$ versus $594.8 \pm 52.2 \mu^{2}$ (si-Irm-1 versus si-scramble, $P<0.05$; Fig. 4h). Altogether, these results demonstrated that the loss of Irm caused a significant delay in injuryinduced muscle regeneration in vivo.

\section{Irm directly interacts with MEF2D}

Next, we probed into the molecular mechanisms underlying the promoting role of Irm in myogenesis. First, RNA-FISH assays in differentiating $\mathrm{C} 2 \mathrm{C} 12$ cells showed that $\mathrm{Irm}$ was distributed in the nucleus, as distinct from the cytoplasmic transcript GAPDH, which was mainly found in the cytoplasm (Fig. 5a). Consistently, Irm was also found in nuclear extracts by performing cellular fractionation assay (Fig. 5b). 


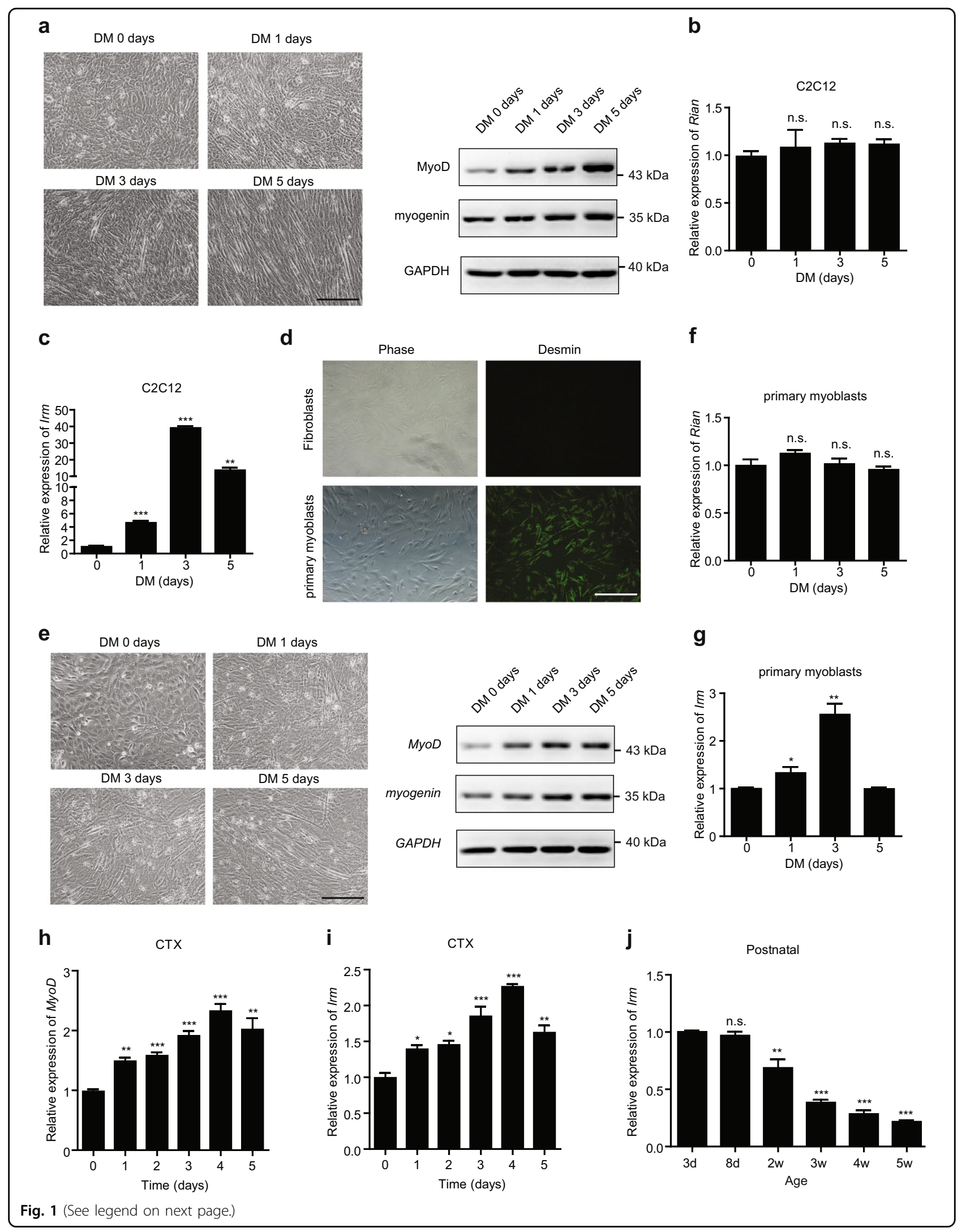


(see figure on previous page)

Fig. $1 \mathrm{lrm}$ is a myogenesis relevant IncRNA. a Left: the representative pictures of $\mathrm{C} 2 \mathrm{C} 12$ cells at $0,1,3$, and 5 days in differentiation medium; right: the protein levels of MyoD and myogenin were detected by western blotting in C2C12 cells at 0, 1, 3, and 5 days in DM. Scale bars, $50 \mathrm{~mm}$. b, c The expression levels of Rian (b) and Irm (c) were detected by qRT-PCR in C2C12 cells at $0,1,3$, and 5 days in DM. $\mathbf{d}$ The certification of the primary myoblasts freshly isolated from mouse limb muscles. Scale bars, $50 \mathrm{~mm}$. e Left: the representative pictures of the primary myoblasts at 0, 1, 3, and 5 days in DM; right: the protein levels of MyoD and myogenin were detected by western blotting in primary myoblasts at 0, 1, 3, and 5 days in DM. Scale bars, $50 \mathrm{~mm}$. $\mathbf{f}, \mathbf{g}$ The expression levels of Rian $(\mathbf{f})$ and $/ \mathrm{rm}(\mathbf{g})$ were detected by qRT-PCR in primary myoblasts at $0,1,3$, and 5 days in DM. $\mathbf{h}$, $\mathbf{i}$ The mRNA levels of MyoD (h) and Irm (i) were detected by qRT-PCR during CTX-induced regeneration. $\mathbf{j}$ The expression levels of Irm were detected by qRT-PCR in muscles of postnatal mice. Data shown represent the mean \pm SEM of three independent experiments. n.S., not significant; ${ }^{*} P<0.05$, ${ }^{* *} P<0.05$, and ${ }^{* * *} P<0.05$ by Student's $t$ test. CTX, cardiotoxin; DM, differentiation medium; GRT-PCR, quantitative real-time polymerase chain reaction

Many nuclear lncRNAs perform their functions through their interaction with proteins ${ }^{18}$. Therefore, to identify the proteins that are associated with Irm, biotin RNA pulldown assay was performed (Fig. 5c). The biotinylated Irm and biotinylated antisense Irm were transcribed in vitro and incubated with whole $\mathrm{C} 2 \mathrm{C} 12$ cell lysates. Biotinlabeled transcripts and their associated cellular proteins were captured by streptavidin beads and then subjected to sodium dodecyl sulfate-polyacrylamide gel electrophoresis (SDS-PAGE) analysis. The same protein samples were then subjected to mass spectrometry analysis, and the proteins specifically binding to Irm were identified (Supplementary Table S2). Among them, MEF2D is a member of the MEF2 family of transcription factors, which act as key mediators of signal-dependent transcription in many different cell types to control developmental processes such as differentiation ${ }^{19}$. Therefore, we selected MEF2D for subsequent validation. First, the association of Irm with MEF2D was validated by immunoblotting assay, and the results showed that MEF2D was clearly detected in Irm-pull-down protein complexes, but not in the complexes associated with antisense Irm (Fig. 5d). To further validate this interaction between Irm and MEF2D, we carried out RNA immunoprecipitation (RIP) of $\mathrm{C} 2 \mathrm{C} 12$ cell nuclear extracts (Fig. 5e). Reverse transcription-polymerase chain reaction (RT-PCR) and qRT-PCR analysis of antibody-enriched RNA revealed that MEF2D antibody pulled down significantly more Irm lncRNA than the IgG control (Fig. 5f).

Next, we sought to determine which region of the Irm transcript mediates its interaction with the MEF2D protein. We generated a series of Irm-deletion probes, which were biotinylated and utilized for RNA pull-down assay. We found that RNA probes containing 1771-2361 nts of the Irm gene, but not others, were capable of pulling down MEF2D, suggesting that the last 590 base pairs of Irm are necessary and sufficient to bind to MEF2D protein (Fig. 5g). Collectively, these findings showed that Irm directly interacts with MEF2D.

\section{Irm regulates MyoD/MEF2D transcriptional activity by binding to MEF2D}

Myogenesis is determined by the master transcriptional regulatory factor MyoD (myogenic differentiation) in concert with other myogenic regulatory factors, such as the MEF2 family members ${ }^{20}$. Given that Irm directly interacts with MEF2D, we speculated that Irm may regulate the transcriptional activity of MyoD/MEF2D.

To this end, we first investigated the effect of Irm on the expression of MyoD/MEF2D target genes. By performing qRT-PCR assay, we found that overexpression of Irm significantly increased the expression of myogenin and miR-206 (Fig. 6a), which are the targets of MyoD/MEF2D. Meanwhile, the knockdown of MEF2D significantly blocked Irm-induced myogenin and miR-206 upregulation in C2C12 cells (Fig. 6a and Supplementary Fig. S1). Consistently, the knockdown of MEF2D significantly inhibited the effect of Irm on the differentiation of $\mathrm{C} 2 \mathrm{C} 12$ cells (Fig. 6b), suggesting that Irm regulates $\mathrm{C} 2 \mathrm{C} 12$ cell differentiation and the expression of myogenin and miR206 in an MEF2D-dependent manner. Next, we wanted to determine whether Irm plays a role in MyoD/MEF2D localization at the promoters of myogenin and miR-206. Luciferase reporter assays using myogenin and miR-206 luciferase reporters revealed inhibited activities with $\mathrm{Irm}$ reduction (Fig. 6c). Consistently, the overexpression of Irm had completely opposite effects in $\mathrm{C} 2 \mathrm{C} 12$ cells (Fig. 6d). Chromatin immunoprecipitation (ChIP)-PCR assays revealed that Irm knockdown caused a significant reduction in the strength of MEF2D binding at the promoters of myogenin and miR-206 (Fig. 6e). Moreover, in the reduction of localization of MEF2D at the appropriate site, the association of MyoD at the promoter regions of myogenin and miR-206 was notably diminished (Fig. 6f). The chromatin isolation by RNA purification assay revealed that Irm directly bound to myogenin and miR -206 promoter regions (Fig. 6g). Taken together, these results strongly suggest that Irm may act as a scaffold lncRNA to recruit MyoD/MEF2D to the promoters of myogenin and miR-206 and thereby transactivate the transcription of myogenin and miR-206, leading to the initiatiation of myogenesis.

\section{Discussion}

Skeletal myogenesis is tightly regulated by a complex network. Accumulating evidence suggests that lncRNAs play a significant role in the regulation of myogenesis ${ }^{21}$. 


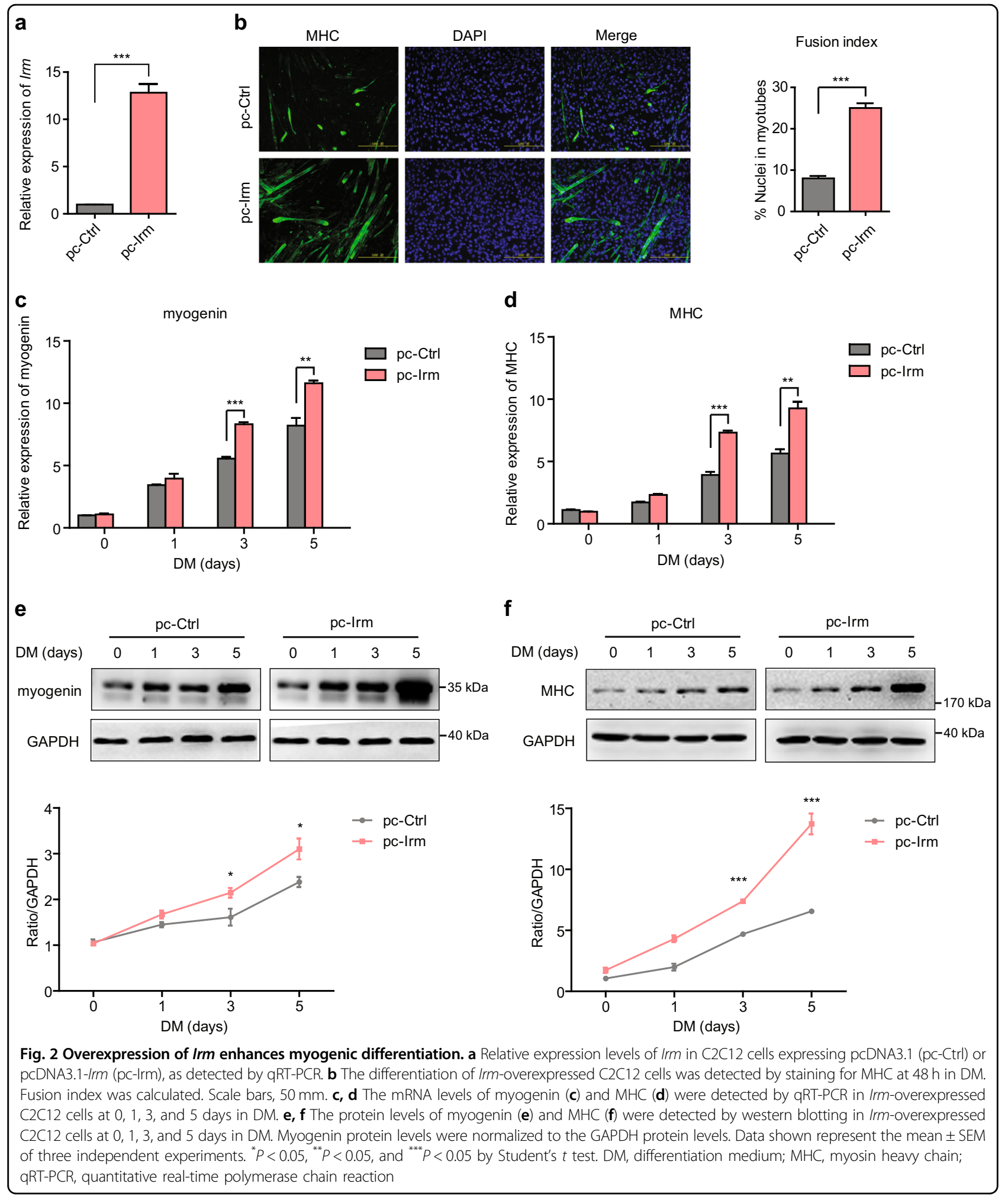

Despite the rapidly increasing number of lncRNAs functionally investigated so far, only a few lncRNAs have been identified to play a role in myogenesis ${ }^{22-25}$. In this study, we reported that IncRNA Irm is upregulated during myogenesis. Functional analyses show that the overexpression of Irm enhances myogenic differentiation, whereas the inhibition of Irm had a completely opposite effect in vitro. Notably, the inhibition of Irm blocked 


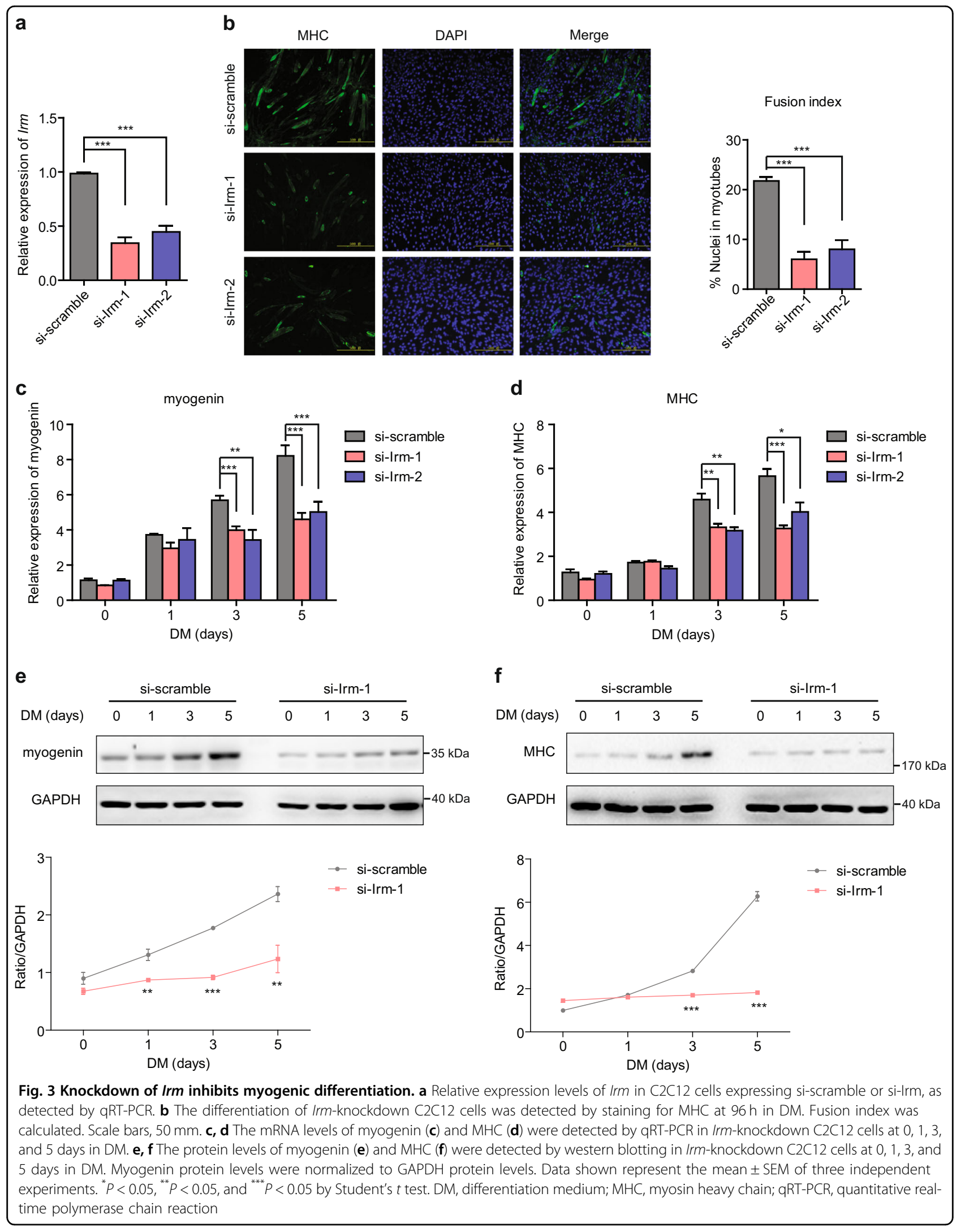




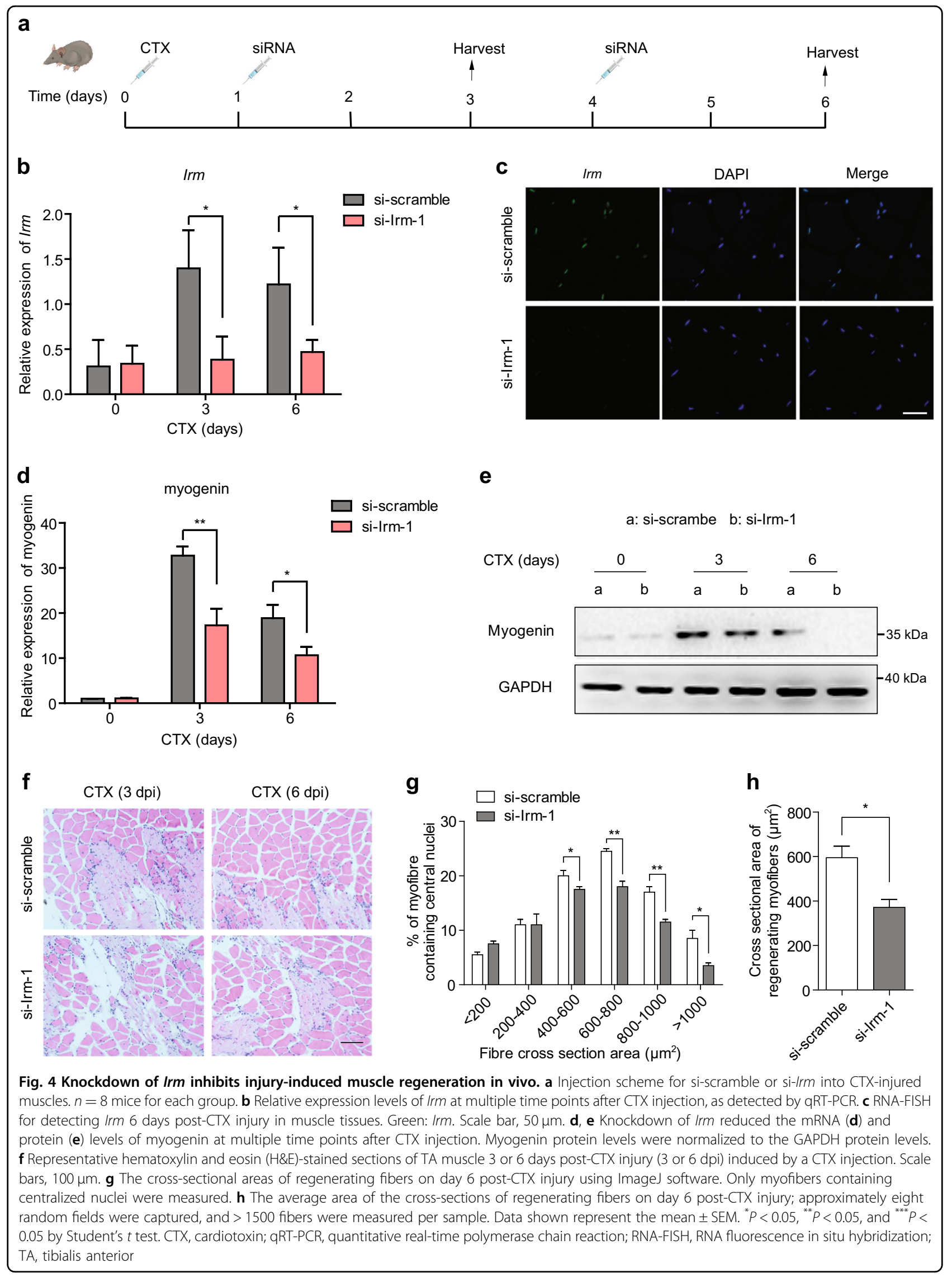




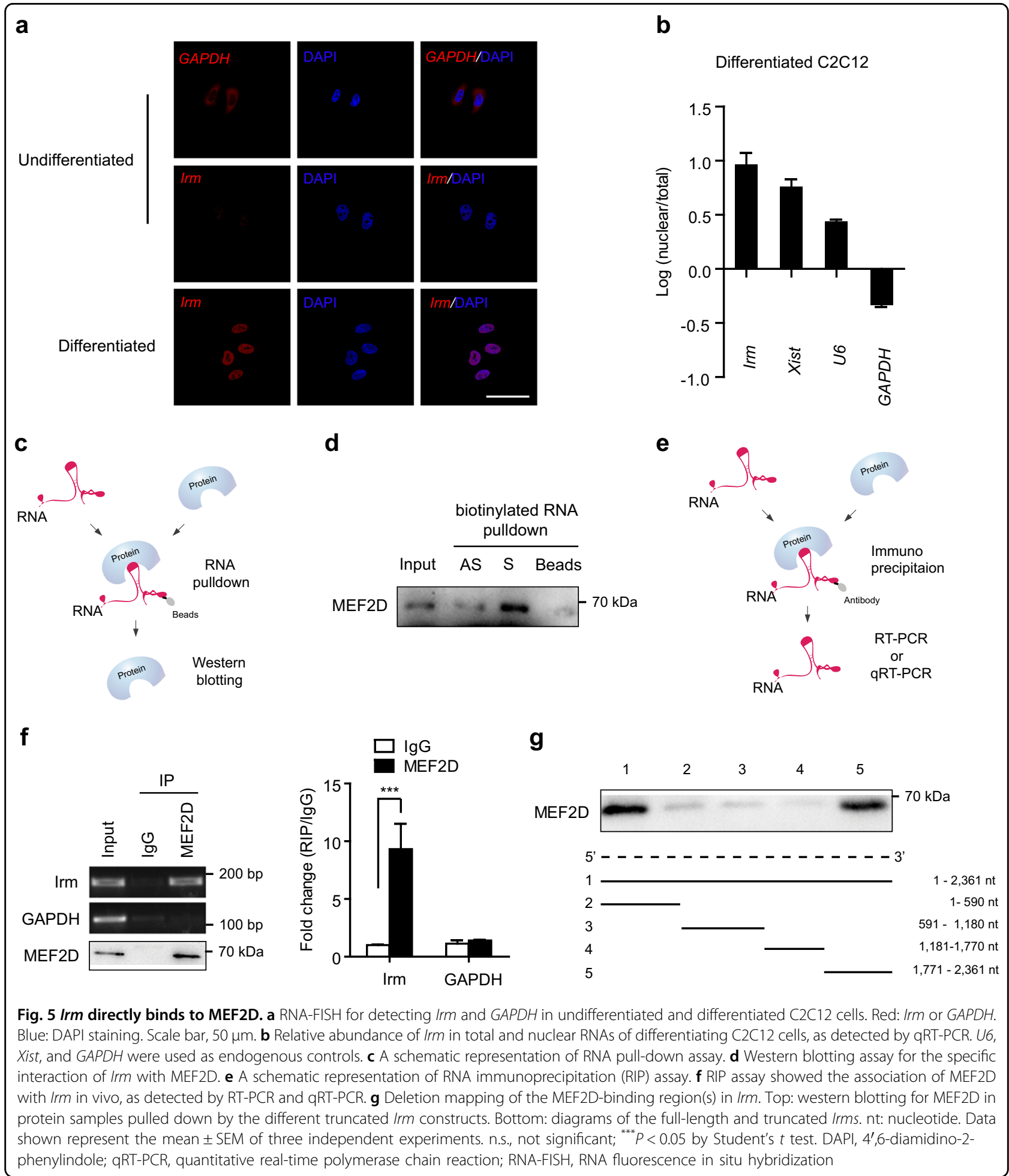

damage-induced muscle regeneration in vivo. Mechanistically, Irm regulated the expression of myogenic genes by directly binding to MEF2D, which in turn promoted the assembly of MyoD/MEF2D on the regulatory elements of target genes. To the best of our knowledge, these findings supported that Irm is a novel player in
MEF2D-regulating network and can promote $\mathrm{C} 2 \mathrm{C} 12$ cell differentiation and myogenesis progression.

Recently, emerging reports are beginning to identify lncRNAs that regulate myogenesis, such as $H 19^{26}, B C 1^{27}$, and MYOSLID ${ }^{28}$. LncRNA Irm was previously identified as an alternatively splicing isoform of Rian gene ${ }^{4}$. Worth 


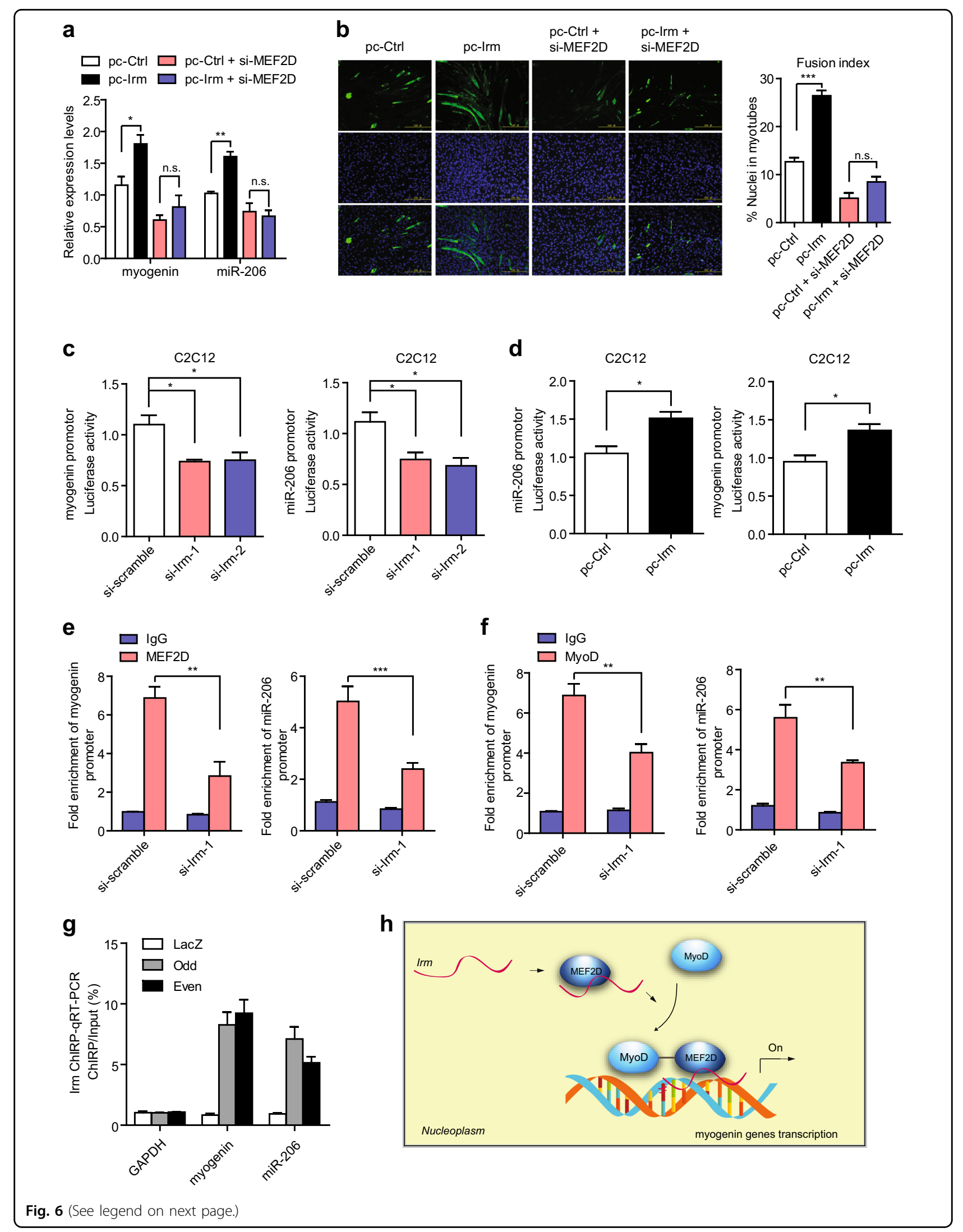


(see figure on previous page)

Fig. 6 Irm enhances the transcriptional activity of MyoD/MEF2D. a The effect of Irm knockdown on the expression of myogenin and miR-206 depended on MEF2D, which was detected by qRT-PCR. b The effect of Irm knockdown on the differentiation of C2C12 cells depended on MEF2D. Fusion index was calculated. Scale bars, $50 \mathrm{~mm}$. c C2C12 cells were transfected with si-Irm or si-scramble, and the luciferase reporter plasmids were generated by inserting the promoter region of myogenin or miR-206. The luciferase activities were measured $48 \mathrm{~h}$ after differentiation. $\mathbf{d} \mathrm{C2C} 12 \mathrm{Cells}$ were transfected with pc-Irm or pc-Ctrl, and the luciferase reporter plasmids were generated by inserting the promoter region of myogenin or miR206. The luciferase activities were measured $48 \mathrm{~h}$ after differentiation. e Knockdown of Irm impaired the binding ability of MEF2D to myogenin and miR-206 promoters, which was determined by ChIP and qRT-PCR assays. $\mathbf{f}$ Knockdown of Irm impaired the binding ability of MyoD to myogenin and miR-206 promoters, which was determined by ChIP and qRT-PCR assays. g Chromatin isolation by RNA purification (ChIRP) assay was performed using even and odd antisense oligos tiling Irm and, a significant amount of genomic DNAs corresponding to myogenin and miR-206 promoters but not in glyceraldehyde 3-phosphate dehydrogenase (GAPDH) locus was retrieved. LacZ ChIRP retrieved no signal. $\mathbf{h}$ Model for Irm-regulating myogenesis. Data shown represent the mean \pm SEM of three independent experiments. n.S., not significant; ${ }^{*} P<0.05,{ }^{* * *} P<0.05$, and ${ }^{* * *} P<0.05$ by Student's $t$ test. ChIP, chromatin immunoprecipitation; qRT-PCR, quantitative real-time polymerase chain reaction

to note, a recent study has suggested that Irm displayed increased expression in tongue and skeletal muscles during embryonic development ${ }^{17}$, indicating that Irm may play a pivotal role in skeletal myogenesis. Indeed, the expression of Irm was significantly increased in differentiated muscle cells, indicating that Irm is associated with active myogenesis. Furthermore, our results showed that Irm significantly promotes the differentiation of $\mathrm{C} 2 \mathrm{C} 12$ cells by performing gain or loss functional assays. Consistently, the loss of Irm caused a significant delay in injury-induced muscle regeneration in vivo. Our study provides a comprehensive characterization of Irm functionally. These findings demonstrated its important regulatory function in skeletal myogenesis.

MyoD and MEF2 families of transcription factors play a prominent role at virtually every step in the skeletal muscle development pathway ${ }^{10,29}$. These two classes of transcription factors interact directly to establish a unique transcriptional regulation network for skeletal muscle gene activation and also to regulate the expression of one another in mutually reinforcing regulatory circuits $^{30}$. Several lncRNAs with enhancer functions in the regulation of transcriptional factor activity have been reported, including $P R-\ln C R N A-1^{31}, E v f-2^{32}, M E G 3^{33}$, and enhancer $\mathrm{RNAs}^{34}$. Interestingly, the expression of MyoD/MEF2D target genes was significantly increased by $\mathrm{Irm}$ in $\mathrm{C} 2 \mathrm{C} 12$ cells, suggesting that Irm may enhance the transcriptional activity of MyoD/MEF2D. Furthermore, ChIP assay showed that the knockdown of Irm significantly inhibited the binding of MyoD/MEF2D to the promoters of their target genes. Mechanistically, Irm directly binds to MEF2D and the DNA of the promoter region to recruit $\mathrm{MyoD} / \mathrm{MEF} 2 \mathrm{D}$ to cause the known target gene activation. Our data support the notion that Irm acts as an IncRNA enhancer of MyoD/MEF2D and synergistically regulates the transcription of myogenic genes in concert with MyoD/MEF2D to mediate myogenic differentiation.

In reviewing the results of this study, a potential limitation should be kept in mind. Although our results have shown that the effects of Irm on $\mathrm{C} 2 \mathrm{C} 12$ cell differentiation in vitro depend on MEF2D, whether the effects of Irm on muscle regeneration in vivo depend on MEF2D is still unclear. It has been proved that MEF2D plays essential roles in muscle differentiation ${ }^{35,} 36$. However, a recent study has shown that the loss of MEF2D in mice has no effect on muscle regeneration in response to CTX injury $^{37}$. These evidences suggested that the function of $\mathrm{Irm} / \mathrm{MEF} 2 \mathrm{D}$ axis in muscle regeneration in vivo is complex and confused, which deserves further investigation in future.

In summary, our results provide a molecular explanation for the Irm-mediated enhancement of MyoD/MEF2D transcriptional activity in myogenic differentiation. Moreover, our findings conceivably indicated that $\mathrm{Irm}$, as a novel regulator of myogenic differentiation, might functionally act as an lncRNA co-activator in guarding the specificity of MyoD/MEF2D transcriptional activity during development.

\section{Materials and methods \\ Cell lines}

Mouse $\mathrm{C} 2 \mathrm{C} 12$ myoblasts were cultured in DMEM supplemented with $10 \%$ fetal bovine serum (FBS), $2 \mathrm{mM}$ L-glutamine, $100 \mathrm{U} / \mathrm{ml}$ penicillin, and $100 \mu \mathrm{g}$ of streptomycin ( $1 \%$ Pen/Strep) at $37^{\circ} \mathrm{C}$ in $5 \% \mathrm{CO}_{2}$. For myogenic differentiation experiment, the cells were seeded in $60 \mathrm{~mm}$ plates, and on reaching 80 90\% confluence, they were shifted to DMEM containing $2 \%$ horse serum.

\section{Primary myoblast isolation}

Total hind limb muscles (eight 10-day-old mice) were digested with $0.25 \%$ type II collagenase (Life Technologies, Carlsbad, CA, USA) and $0.25 \%$ trypsin in $37^{\circ} \mathrm{C}$ for 1 h. Furthermore, the fibroblasts were removed by differential centrifugation. Finally, the primary myoblast cells were certificated by desmin antibody (Santa Cruz Biotechnology, USA) and cultured in F10/DMEM medium (1:1) supplemented with 20\% FBS and basic fibroblast growth factor (Life Technologies; $25 \mathrm{ng} / \mathrm{ml}$ ). 


\section{RNA extraction and qRT-PCR assay}

Total RNAs were extracted from cultured cells or tissues using HiFi RNA Extract kit (Beijing BLKWbio Co., Ltd, China) according to the manufacturer's protocol. The complementary DNAs were synthesized from $500 \mathrm{ng}$ of total RNAs using PrimeScript RT reagent kit (Takara, Dalian, China) according to the manufacturer's protocol. The qRT-PCR assays were performed in the BioRad IQ5 Real-Time PCR System (BioRad, Hercules, CA, USA) using KAPA SYBR ${ }^{\circ}$ FAST qPCR Master Mix (KAPA, Wilmington, UK). The relative expression of RNAs was calculated using the comparative Ct method. The primer sequences are described in Supplementary Table S1. Data represent the mean \pm SEM of three independent experiments.

\section{Overexpression of Irm}

For Irm overexpression, the Irm sequences were cloned into pcDNA3.1 vector $(+)$ (Invitrogen, USA). The constructs were confirmed by DNA sequencing. The vectors with an intact sequence of Irm were transfected into mouse C2C12 myoblasts using Lipofectamine 2000 (11668-019; Invitrogen, USA), following the manufacturer's instructions. All media contained 10\% FBS, $100 \mathrm{U} / \mathrm{ml}$ penicillin, and $100 \mathrm{mg} / \mathrm{ml}$ streptomycin (15140-122; Gibco, USA). Cells were incubated in humidified incubators equilibrated with $5 \% \mathrm{CO}_{2}$ at $37^{\circ} \mathrm{C}$.

\section{RNA interference}

siRNAs against Irm were synthesized by GenePharma (Shanghai city, China). The sequences of siRNAs used against Irm were the following: siRNA-1, 5'-GCACACT ACTGTTGAATTA-3' and siRNA-2, 5'-GCAGGATTCA GATAGTAAT-3'. The sequence of siRNA used against $M e f 2 d$ was siRNA, $5^{\prime}$-GAGGCAAAGGGTTAATGCATC ATTT-3'. For siRNA transfection, the cells were transfected with the indicated siRNAs using Lipofectamine 2000 (11668-019; Invitrogen, USA), following the manufacturer's instructions.

\section{Immunofluorescence assay}

C2C12 cells were washed twice in $1 \times$ phosphate-buffered saline (PBS), fixed with $4 \%$ paraformaldehyde for $30 \mathrm{~min}$, permeabilized with $0.5 \%$ Triton X-100-PBS for $5 \mathrm{~min}$, and then blocked for $30 \mathrm{~min}$ with $3 \%$ bovine serum albumin/ $0.2 \%$ Triton X-100-PBS. To detect endogenous MHC, cells were incubated with primary rat monoclonal anti-MHC (1:200; Santa Cruz Biotechnology, USA) for $12 \mathrm{~h}$ at $4{ }^{\circ} \mathrm{C}$. Secondary antibodies were conjugated with Alexa488labeled secondary antibody (Invitrogen, USA) and used in a 1:1000 dilution for $2 \mathrm{~h}$ at $37^{\circ} \mathrm{C}$. 4',6-Diamidino-2-phenylindole was added for $5 \mathrm{~min}$ in the dark before taking images with a confocal microscope (ZEISS, LSM880 + ELYRAS.1). Eight micrographs per field were performed.

\section{Animal studies}

C57BL/6 mice were purchased from Liaoning Changsheng Biological Technology Company and maintained in the Institute of Special Economic Animal and Plant Science of CAAS Institutions at a constant temperature and humidity with a standard diet. C57B/L mice were housed in animal care facilities for at least 15 days before experiment. Our works were approved by the Animal Care Committee of the Institute of Special Economic Animal and Plant Science of CAAS and were conducted in accordance with the CAAS Guide for the Care and Use of Laboratory Animals. For CTX (Latoxan, Valence, France) injection, a muscle injury was induced in mice by injecting CTX ( $50 \mathrm{ml}$ of $10 \mu \mathrm{M}$ CTX in PBS) into the right tibialis anterior muscles. Oligos were prepared by preincubating $20 \mu \mathrm{m}$ siRNA oligos with Lipofectamine 2000 for $15 \mathrm{~min}$ and injections were made in a volume of $100 \mu \mathrm{l}$ minimum essential medium (Invitrogen, NY, USA) on days 1 and 4. Eight mice were used in each group. The muscles were harvested on days 0,3 , and 6 , and total RNAs and proteins were extracted for real-time RT-PCR and western blotting assays. Mice were killed by $\mathrm{CO}_{2}$, and all efforts were made to minimize suffering.

\section{Hematoxylin and eosin stainings}

Fresh isolated tibialis anterior muscle samples were mounted on $4 \%$ paraformaldehyde. The sections of muscle tissues $(10 \mu \mathrm{m})$ were subjected to hematoxylin and eosin staining using a commercial kit (Solarbio, China). The sections were detected using OLYMPUS Digital Pathology (OLYMPUS, Japan). Eight micrographs per field were performed for the photomicrographs.

\section{Western blotting analysis}

For the protein analysis of whole-cell lysates, cells were lysed in radioimmunoprecipitation assay (CWBIO, China) buffer plus ethylenediaminetetraacetic acid-free protease inhibitor cocktail (04693132001; Roche). Total proteins $(10-20 \mu \mathrm{g})$ were re-suspended in Laemmli buffer $(63 \mathrm{mM}$ Tris- $\mathrm{HCl}, 10 \%$ glycerol, $2 \%$ SDS, $0.0025 \%$ bromophenol blue (pH 6.8)) and electrophoresed on SDS-polyacrylamide gels. Then, the proteins were transferred to polyvinylidene difluoride membranes. Membranes were incubated with the indicated primary antibodies and anti-mouse or anti-rabbit secondary antibodies conjugated to horseradish peroxidase. The immunoreactive bands were detected using SuperSignal $^{\text {Tw }}$ West Pico Chemiluminescent Substrate kit (Thermo Fisher Scientific, USA) and Western Blotting Detection System (BioRad, USA). To detect the protein levels of GAPDH, MHC, and myogenin, anti-GAPDH (1:5000; Santa Cruz Biotechnology, USA), anti-MHC (1:1000; Santa Cruz Biotechnology, USA), and antimyogenin (1:2000; Santa Cruz Biotechnology, USA) antibodies were used. 


\section{RNA fluorescence in situ hybridization}

RNA-FISH analyses in $\mathrm{C} 2 \mathrm{C} 12$ cells and muscle tissues were performed as described previously ${ }^{38}$. The RNA probes were prepared using the DIG RNA Labeling Kit (SP6/T7). PCR primers for RNA probe synthesis are the following: F, 5'-GCACACTACTGTTGAATTA-3' and R, 5'-GCAGGATTCAGATAGTAAT-3'. The digoxigenin haptens were detected by fluorescein-conjugated antiDIG Fab fragment (1:200; Roche, USA). The nuclei were stained with 4',6-diamidino-2-phenylindole. The signals were observed under a confocal microscope (ZEISS, LSM880 + ELYRAS.1).

\section{Subcellular localization assay}

RNAs were extracted from the nuclei and cytoplasm (total) or only nuclei (nuclear) of C2C12 cells using PARIS kit (Invitrogen) according to the manufacturer's protocol. One microgram of RNA extracted from total and nuclear fractions, respectively, was used for qRT-PCR analyses of Irm, Xist, U6 RNA (nuclear retained), and GAPDH mRNA (exported to the cytoplasm). Data represent the mean \pm SEM of three independent experiments.

\section{RNA pull-down assay}

RNA pull down was performed as previously described $^{39}$. In brief, the pGEM-T vectors (Promega, Madison, WI, USA) carrying Irm, antisense of Irm, truncated fragments of Irm or GAPDH DNAs were linearized with the corresponding restriction enzymes to prepare the template DNAs for in vitro transcription. Then, biotin-labeled RNA transcripts were transcribed in vitro using T7 RNA polymerase (Roche, Basle, Switzerland). Three micrograms of in vitro biotinylated RNAs were mixed with proteins extracted from $\mathrm{C} 2 \mathrm{C} 12$ cells, followed by targeting the RNAs with streptavidin beads (Millipore, Bedford, MA). The coprecipitated proteins were then visualized by western blotting.

\section{RNA immunoprecipitation assay}

We performed RIP experiments using Magna RIP RNABinding Protein Immunoprecipitation Kit (Millipore, Bedford, MA, USA) according to the manufacturer's instructions. The antibody against MEF2D (Santa Cruz Biotechnology, USA) was used for RIP. The coprecipitated RNAs were detected by qRT-PCR assay. Total RNAs (input controls) and isotype controls were assayed simultaneously to demonstrate that the detected signals were from RNAs specifically binding to MEF2D. Data represent the mean \pm SEM of three independent experiments.

\section{Luciferase reporter assay}

For luciferase reporter assay, reporter plasmids with the promoter region of myogenin or miR-206 were transfected into $\mathrm{C} 2 \mathrm{C} 12$ cells by Lipofectamine 2000 (Invitrogen, USA) in 96-well plates. The luciferase activities were measured $48 \mathrm{~h}$ after differentiation by using Dual-Luciferase Reporter Assay System (Promega, Madison, WI, USA). Firefly activity was normalized to Renilla luciferase activity. Data represent the mean \pm SEM of three independent experiments.

\section{Chromatin immunoprecipitation}

MyoD and MEF2D ChIP was performed with Magna $\mathrm{ChIP}^{\mathrm{m} \mathrm{s}} \mathrm{A} / \mathrm{G}$ One-Day Chromatin Immunoprecipitation Kits (Millipore, Bedford, MA) according to the manufacturer's instructions. Briefly, the ChIPed DNA was eluted, reverse X-linked, purified, and analyzed by qRTPCR. Data represent the mean \pm SEM of three independent experiments.

\section{Chromatin isolation by RNA purification}

Chromatin isolation by RNA purification was performed as previously described ${ }^{40}$. The antisense probes (20 nucleotides in length) were designed and divided into odd and even pools. Oligonucleotides were biotinylated at the $3^{\prime}$ end with an 18-carbon spacer arm. First, C2C12 cells, which were harvested, were cross-linked and sonicated. Cell lysates and the probes were then incubated at $37^{\circ} \mathrm{C}$ for $6 \mathrm{~h}$. Furthermore, magnetic streptavidin beads were added to pull-down probes and separated with a magnet strip. DNA isolated by phenol-chloroform-ethanol precipitation was used for qRT-PCR. DNA-binding enrichment was represented by the percentage of pull-down DNA over input DNA. Data represent the mean \pm SEM of three independent experiments.

\section{Statistical analysis}

All statistical analyses were performed using GraphPad Prism 5 software. All values are expressed as the mean \pm SEM of three independent experiments. Student's $t$ test was used for statistical analyses. $P$ values lower than 0.05 were considered to be statistically significant.

\footnotetext{
Acknowledgements

We are grateful to the Beijing Proteome Research Center and Beijing Institute of Radiation Medicine for their assistance in digital microscopy. We thank Dr. Qin for helpful discussions. This study was funded by the Science and Technology Innovation Project of Chinese Academy of Agricultural Sciences grant CAAS-ASTIP-2016-ISAPS. We thank the anonymous reviewers for their helpful insights and comments regarding this manuscript. This study was designed by G.Y.L. The manuscript was written by Y.T.S. The experiments were performed and analyzed by Y.T.S., Y.H, and X.Y.Z.
}

Conflict of interest

The authors declare that they have no conflict of interest.

Publisher's note

Springer Nature remains neutral with regard to jurisdictional claims in published maps and institutional affiliations. 
Supplementary Information accompanies this paper at (https://doi.org/ 10.1038/s41419-019-1399-2).

Received: 22 August 2018 Revised: 20 December 2018 Accepted: 4 January 2019

Published online: 21 February 2019

\section{References}

1. Zhang, S. et al. m(6)A demethylase ALKBH5 maintains tumorigenicity of glioblastoma stem-like cells by sustaining FOXM1 expression and cell proliferation program. Cancer Cell. 31, 591-606 e596 (2017).

2. Jiang, R. et al. The long noncoding RNA Inc-EGFR stimulates T-regulatory cells differentiation thus promoting hepatocellular carcinoma immune evasion. Nat. Commun. 8, 15129 (2017).

3. Mueller, A. C. et al. MUNC, a long noncoding RNA that facilitates the function of MyoD in skeletal myogenesis. Mol. Cell. Biol. 35, 498-513 (2015).

4. Wallace, C. et al. The imprinted DLK1-MEG3 gene region on chromosome $14 \mathrm{q} 32.2$ alters susceptibility to type 1 diabetes. Nat. Genet. 42 68-71 (2010).

5. Hu, W. L. et al. GUARDIN is a p53-responsive long non-coding RNA that is essential for genomic stability. Nat. Cell Biol. 20, 492-502 (2018).

6. Liang, T. et al. InCRNA AK017368 promotes proliferation and suppresses differentiation of myoblasts in skeletal muscle development by attenuating the function of miR-30c. FASEB J. 32, 377-389 (2018).

7. Wang, L. et al. LncRNA Dum interacts with Dnmts to regulate Dppa2 expression during myogenic differentiation and muscle regeneration. Cell Res. 25, 335-350 (2015).

8. Zhu, M. et al. Lnc-mg is a long non-coding RNA that promotes myogenesis. Nat. Commun. 8, 14718 (2017).

9. Puri, P. L. \& Sartorelli, V. Regulation of muscle regulatory factors by DNAbinding, interacting proteins, and post-transcriptional modifications. J. Cell. Physiol. 185, 155-173 (2000).

10. Berkes, C. A. \& Tapscott, S. J. MyoD and the transcriptional control of myogenesis. Semin. Cell. Dev. Biol. 16, 585-595 (2005)

11. Tapscott, S. J. The circuitry of a master switch: Myod and the regulation of skeletal muscle gene transcription. Development 132, 2685-2695 (2005).

12. Bonasio, R. \& Shiekhattar, R. Regulation of transcription by long noncoding RNAs. Annu. Rev. Genet. 48, 433-455 (2014).

13. Legnini, I., Morlando, M., Mangiavacchi, A., Fatica, A. \& Bozzoni, I. A feedforward regulatory loop between HuR and the long noncoding RNA linc-MD1 controls early phases of myogenesis. Mol. Cell 53, 506-514 (2014).

14. $Y u, X$. et al. Long non-coding RNA Linc-RAM enhances myogenic differentiation by interacting with MyoD. Nat. Commun. 8, 14016 (2017).

15. Zhou, L. et al. Linc-YY1 promotes myogenic differentiation and muscle regeneration through an interaction with the transcription factor $\mathrm{YY1}$. Nat. Commun. 6, 10026 (2015).

16. Cavaille, J., Seitz, H., Paulsen, M., Ferguson-Smith, A. C. \& Bachellerie, J. P. Identification of tandemly-repeated C/D snoRNA genes at the imprinted human $14 \mathrm{q} 32$ domain reminiscent of those at the Prader-Willi/Angelman syndrome region. Hum. Mol. Genet. 11, 1527-1538 (2002).

17. Gu, T. et al. Expression of macro non-coding RNAs Meg8 and Irm in mouse embryonic development. Acta Histochem. 114, 392-399 (2012).

18. Huarte, M. et al. A large intergenic noncoding RNA induced by p53 mediates global gene repression in the p53 response. Cell 142, 409-419 (2010).
19. Naya, F. J. \& Olson, E. MEF2: a transcriptional target for signaling pathways controlling skeletal muscle growth and differentiation. Curr. Opin. Cell Biol. 11, 683-688 (1999).

20. Singh, K. et al. A KAP1 phosphorylation switch controls MyoD function during skeletal muscle differentiation. Genes Dev. 29, 513-525 (2015).

21. Li, Y., Chen, X., Sun, H. \& Wang, H. Long non-coding RNAs in the regulation of skeletal myogenesis and muscle diseases. Cancer Lett. 417, 58-64 (2018).

22. Militello G. et al. A novel long non-coding RNA Myolinc regulates myogenesis through TDP-43 and Filip1. J. Mol. Cell Biol. 10, 102-117 (2018).

23. Zhang Z. K. et al. A newly identified IncRNA MAR1 acts as a miR-487b sponge to promote skeletal muscle differentiation and regeneration. J. Cachexia Sarcopenia Muscle 9, 613-626 (2018).

24. $\mathrm{Xu}, \mathrm{X}$. et al. LncRNA H19 promotes the differentiation of bovine skeletal muscle satellite cells by suppressing Sirt1/FoxO1. Cell. Mol. Biol. Lett. 22, 10 (2017).

25. Han, X., Yang, F., Cao, H. \& Liang, Z. Malat1 regulates serum response factor through miR-133 as a competing endogenous RNA in myogenesis. FASEB $\mathrm{J}$. 29, 3054-3064 (2015).

26. Dey, B. K., Pfeifer, K. \& Dutta, A. The $\mathrm{H} 19$ long noncoding RNA gives rise to microRNAs miR-675-3p and miR-675-5p to promote skeletal muscle differentiation and regeneration. Genes Dev. 28, 491-501 (2014).

27. Wang, Y. C., Chuang, Y. H., Shao, Q., Chen, J. F. \& Chen, S. Y. Brain cytoplasmic RNA 1 suppresses smooth muscle differentiation and vascular development in mice. J. Biol. Chem. 293, 5668-5678 (2018).

28. Zhao, J. et al. MYOSLID is a novel serum response factor-dependent long noncoding RNA that amplifies the vascular smooth muscle differentiation program. Arterioscler. Thromb. Vasc. Biol. 36, 2088-2099 (2016).

29. Taylor, M. V. \& Hughes, S. M. Mef2 and the skeletal muscle differentiation program. Semin. Cell. Dev. Biol. 72, 33-44 (2017).

30. Black, B. L. \& Olson, E. N. Transcriptional control of muscle development by myocyte enhancer factor-2 (MEF2) proteins. Annu. Rev. Cell. Dev. Biol. 14 167-196 (1998).

31. Li, N. \& Richard, S. Sam68 functions as a transcriptional coactivator of the p53 tumor suppressor. Nucleic Acids Res. 44, 8726-8741 (2016).

32. Feng, J. et al. The Evf- 2 noncoding RNA is transcribed from the Dlx-5/6 ultraconserved region and functions as a Dlx-2 transcriptional coactivator. Genes Dev. 20, 1470-1484 (2006).

33. Zhu, J. et al. Long noncoding RNA MEG3 interacts with p53 protein and regulates partial p53 target genes in hepatoma Cells. PLOS. ONE. 10, e0139790 (2015).

34. Kim, T. K. et al. Widespread transcription at neuronal activity-regulated enhancers. Nature 465, 182-187 (2010).

35. Choi, J. et al. Modulation of lysine methylation in myocyte enhancer factor 2 during skeletal muscle cell differentiation. Nucleic Acids Res. 42, 224-234 (2014).

36. Sebastian, S. et al. Tissue-specific splicing of a ubiquitously expressed transcription factor is essential for muscle differentiation. Genes Dev. 27, 1247-1259 (2013).

37. Liu, N. et al. Requirement of MEF2A, C, and D for skeletal muscle regeneration. Proc. Natl Acad. Sci. USA 111, 4109-4114 (2014).

38. Tabara, H., Motohashi, T. \& Kohara, Y. A multi-well version of in situ hybridization on whole mount embryos of Caenorhabditis elegans. Nucleic Acids Res. 24, 2119-2124 (1996)

39. Tsai, M. C. et al. Long noncoding RNA as modular scaffold of histone modification complexes. Science 329, 689-693 (2010).

40. Chu C., Quinn J., \& Chang H. Y. Chromatin isolation by RNA purification (ChIRP). J. Vis. Exp. 61, 1-6 (2012). 